Cambouis, la revue des sciences sociales aux mains sales

\title{
Le répertoire figuratif des personnes interrogées
}

\section{François de Singly}

Professeur à l'université de Rennes II

Résumé: L'univers professionnel dans lequel vivent sociologues et statisticiens les conduit à oublier fréquemment que les intérêts scientifiques auxquels ils adhèrent ne constituent pas un intérêt général et que leurs intérêts peuvent déranger d'autres intérêts particuliers. On voudrait faire ici le relevé des modalités par lesquelles les individus (et les groupes) se protègent de l'enquête et étudier les effets de cette résistance sur le matériel recueilli. Cette communication propose une extension de la problématique d'Erving Goffman, notamment celle des Rites d'interaction (Goffman, 1974), à la situation de l'interrogatoire scientifique. Elle décrit le travail de figuration auquel sont contraints les individus soumis au questionnement. Ceux-ci doivent utiliser au mieux leur compétence théâtrale, requise dans toute interaction, pour éviter les faux pas plus probables étant donné certaines caractéristiques de la situation d'enquête et de la structure du questionnaire.

Date de publication : 8/7/2021

Dossier : Sociologie et statistique

Comment citer : $10.52983 /$ crev.vi0.37

Licence:Cambouis publie ses contenus selon les termes de la Licence Creative Commons Attribution - Pas d'Utilisation Commerciale - Pas de Modification 4.0 International. Les auteurices gardent leurs droits de propriété intellectuelle pleine et entière sur leurs articles. 


\section{Le répertoire figuratif des personnes interrogées}

\section{François de Singly}

Professeur à l'université de Rennes II

« Il se pose la question de l'opportunité des réponses à donner aux questions qu'il pose, mais il ne la résout pas, crainte de rien précipiter » Robert Pinget, Monsieur Songe

L'univers professionnel dans lequel vivent sociologues et statisticiens les conduit à oublier fréquemment que les intérêts scientifiques auxquels ils adhèrent ne constituent pas un intérêt général et que leurs intérêts peuvent déranger d'autres intérêts particuliers. Jeanne Favret-Saada dans Corps pour corps (Favret-Saada, 1981) raconte comment elle a perçu ce conflit lors d'une enquête sur la sorcellerie dans le bocage. Les refus de lui donner de l'information sur les sorts prennent une forme polie et déguisée puisqu'elle s'est installée au «pays » et se conduit comme il faut. On ne lui tourne pas le dos, c'est quelqu'un «à qui on peut parler, mais à qui l'on parle de riens : des rapports entre généalogies des chats et des humains, de la recette de cuisson des renards, de la santé des enfants ou de leur bonne éducation » (ibid., p. 41). Les mois passent et l'ethnologue n'arrive pas à obtenir un discours commençant par un «moi, je crois dans les sorts », ce sont en effet toujours «les autres qui croient que » (ibid, $p$. 32). Ce mutisme est doublement protecteur : d'une part, la personne interrogée n'est pas classée dans le groupe des adhérents à une croyance « ridicule, infantile, abracadabrante» (ibid., p. 32), d'autre part, elle préserve son avenir - «moins on en parle, moins on est pris » (ibid., p. 98).

Le mutisme ne constitue qu'une des formes de la résistance au questionnement. On voudrait faire ici le relevé des modalités par lesquelles les individus (et les groupes) se protègent de l'enquête et étudier les effets de cette résistance sur le matériel recueilli. Cette communication propose une extension de la problématique d'Erving Goffman, notamment celle des Rites d'interaction (Goffman, 1974), à la situation de l'interrogatoire scientifique.

Elle décrit le travail de figuration auquel sont contraints les individus soumis au questionnement. Ceux-ci doivent utiliser au mieux leur compétence théâtrale, requise dans toute interaction, pour éviter les faux pas plus probables étant donné certaines caractéristiques de la situation d'enquête et de la structure du questionnaire. 


\section{Faire bonne figure lors de l'interrogatoire}

Comme dans toute relation, la personne interrogée essaie de garder la face, c'est-à-dire de revendiquer une valeur sociale positive à travers la ligne de conduite, explicite ou non, qu'elle s'est fixée pour la durée du contact avec l'enquêteur.

Cela implique que l'individu enquêté assure un certain «maintien de l'ordre expressif » pour que rien durant l'interaction ne vienne contredire ou ternir l'image qu'il veut donner de lui-même. La relation d'enquête impose aux personnes interrogées cette alternative inhérente à toute interaction sociale : comment faire bonne figure ou perdre la face?

Certaines modalités, par lesquelles la personne interrogée se met en scène, ne peuvent être comprises que si on saisit que la situation d'enquête n'est pas réductible à une interaction entre un enquêteur et un enquêté. À la différence de la confession, par exemple, où le péché avoué reste "privé », l'enquête sociologique transforme les territoires de la possession qui sont dévoilés pendant son déroulement ; même avec les garanties de l'anonymat, elle fabrique à partir de ces opinions privées de l'opinion publique ${ }^{1}$. Cette opération publicitaire - dont l'enregistrement des réponses témoigne - complique la tâche de la personne interrogée qui doit, par ses représentations théâtrales, influencer non seulement les représentations mentales de l'enquêteur mais également, à travers lui, celles des personnes et institutions qui forment son public. L'implication personnelle est augmentée par cette publicité. Les psychologues expérimentaux - comme Jean-Pierre Desportes dans Les effets de la présence de l'expérimentateur dans les sciences du comportement (Desportes, 1975) - ont remarqué que les sujets d'une expérience qui sont prévenus de la présence de spectateurs et du statut social de ces spectateurs tendent à percevoir cette présence comme « une menace »; ils craignent que ne soit porté sur eux un jugement négatif.

Le danger - réel ou non - des enquêtes, « présences constantes, attentives, curieuses $»^{2}$, continue à alimenter l'imaginaire des agents sociaux dans la France contemporaine et à susciter des réactions de défense et de défiance, audibles ou non ${ }^{3}$. Les risques liés à la perte de contrôle de ses expressions ne sont pas limités à la dévalorisation de soi auprès d'une seule personne, ils comprennent les menaces concernant ses intérêts matériels et symboliques.
1 Sur « L’Archéologie de la publicité comme dimension constitutive de la société bourgeoise », voir (Habermas, 1978).

2 Pour reprendre les expressions de Michel Foucault dans La volonté de savoir (Foucault, 1976). L'histoire des enquêtes dans les sciences sociales — comme celle de Gérard Leclerc dans L'observation de l'homme (Leclerc, 1979) - montre que l'appareil d'observation des agents sociaux renforce dans son autonomie relative la volonté de savoir des classes dominantes ou de certaines de ses fractions.

3 C omme lors des recensements de la population. Pour exemple, voir l'article de Pringle ( « On nous recense ou on nous espionne », Elle, avril 1982) où le regard inquisiteur de Madame « Marianne-INSEE, Marianne Sans-Gêne » et le « goût pervers des experts en statistiques » sont critiqués.

4 Au contraire, Luc Boltanski et Pascale Maldidier dans $L a$

vulgarisation scientifique et son public (Boltanski et Maldidier, 1977), à propos d'une comparaison entre les structures des lecteurs de Sciences et Vie et celles de ceux qui ont répondu volontairement à une enquête sur leurs lectures, montrent l'intérêt sociologique des échantillons les moins « représentatifs ».

5 Développé notamment par Daniel Gaxie (1978).

\section{Première alternative : évitement ou acceptation}

La première possibilité pour un individu confronté à une interaction dangereuse est la fuite. «Le plus sûr moyen de prévenir le danger est d'éviter les rencontres où il risque de se manifester », remarque Goffman. Le procédé d'évitement de l'enquête - sous les prétextes du manque de temps, de compétence (« il y a des gens mieux informés ») qui permettent à l'individu contacté de «se défiler »-n'apparaît dans les comptes rendus d'enquête qu'en annexe : il est précisé alors que la structure des répondants étant proche de la structure de la population de référence ces défections ont peu d'importance ${ }^{4}$. Les individus réfractaires sont passés dans la colonne «pertes» sans affecter le bilan, ce dernier pouvant être au besoin «redressé ».

L'interprétation de cette procédure d'évitement n'est pas réductible à la grille selon laquelle le silence est expliqué par le couple «incompétence et inintérêt $»^{5}$. Refuser de parler ne signifie pas nécessairement ne rien avoir à dire, ou craindre de dire des erreurs. L'interrogatoire n'est pas seulement comparable à la situation scolaire ; il faut le rapprocher d'un ensemble plus 
vaste d'interactions sociales - les rencontres entre les administrés, l'État et sa bureaucratie. Une partie du public potentiel des enquêtes (qu'une analyse des réponses et des non-réponses à un sondage portant sur le rapport à l'État permet de circonscrire (de Singly, 1982)) déjà réfractaire à toute communication avec les fonctionnaires est encore plus réticente devant les enquêtes statistiques dans la mesure où aucune contrepartie positive ne lui est présentée, sinon l'avenir de la connaissance scientifique ${ }^{6}$.

\section{Seconde alternative : « congruence » ou non}

Le chercheur espère que les individus qu'il interroge ou qu'il fait interroger éviteront de falsifier leurs opinions ou leurs comportements au travers des réponses qu'ils lui donnent ${ }^{7}$.

Le point de vue adopté ici consiste à se situer de l'autre côté, en se mettant à la place des individus interrogés afin de percevoir les limites de leur authenticité. L'authenticité d'un sujet est définie comme la congruence entre son état personnel et l'expression publique de cet état. Prendre ce critère pour analyser la situation d'enquête n'implique pas que la congruence soit pour le sujet interrogé la posture idéale. S'il est vrai qu'il paraît souhaitable pour le chercheur de rencontrer des individus « authentiques », il n'est pas obligatoirement de l'intérêt de ces interrogés de l'être. De nombreux évènements de la vie quotidienne - à commencer par les salutations - n'exigent-ils pas de la part des acteurs sociaux une aptitude à la dissimulation? Harvey Sacks l'a démontré dans «Tout le monde doit mentir» (Sacks, 1973). L'expression authentique de soi comporte des risques, le rappel d'un passé compromettant par exemple - un passage en prison, en hôpital psychiatrique ${ }^{8}$ - pouvant avoir des effets négatifs, stigmatisants. Aussi une personne interrogée peut être amenée, en fonction des représentations qu'elle a de la situation, à donner une représentation frauduleuse d'elle-même pour ne pas avoir à perdre la face devant l'enquêteur et le public qui se profile derrière lui.

La production de la réponse à une question posée dépend donc de l'état personnel de l'individu interrogé et de la manière dont il se représente la situation ${ }^{9}$. Deux cas possibles :

- soit la situation d'enquête semble autoriser l'authenticité du sujet ; dans ce cas, le sujet, si la question posée comprend un éventail de réponses suffisant, choisit la réponse dans le stock des réponses correspondant à son état personnel ;

- soit la situation d'enquête est perçue menaçante par le sujet; dans ce cas, celui-ci sélectionne une réponse qui diffère de son état personnel. $\mathrm{Si}$ la question est une question de connaissance (a), le sujet ignorant la «bonne» réponse doit se déterminer entre deux types de réponses non congruentes : est-il préférable d'apparaître comme ne sachant pas ou comme ayant répondu «à côté »? Si la question est une question
6 Jeanne Favret-Saada note devant la résistance d'un sujet interrogé : « si j'ai bien compris, il voit en moi un représentant de "l'administration", poubelle où il fourre les gendarmes, les juges, les agents du fisc et les employés du Crédit Agricole » (Favret-Saada, 1981, p. 232).

7 Au moment de la publication des résultats, les doutes s'estompent et l'hypothèse de la représentation frauduleuse est oubliée ; tout au moins estime-t-on que la part des « imposteurs » dans l'échantillon est suffisamment faible pour qu'elle ne perturbe pas la moyenne des résultats.

8 Sur la nécessité de la représentation frauduleuse en de tels cas, voir Schwartz et Skolnick (1962).

9 Un recensement des éléments qui contribuent à rendre la situation ou telle partie de la situation insupportable aux sujets interrogés est proposé dans « La gestion sociale des silences » (de Singly, 1982). 
d'opinion (b), le sujet ne voulant pas dévoiler son état personnel doit également se décider entre le fait d'afficher une autre opinion et le fait de déclarer publiquement son refus de répondre ${ }^{10}$.

\section{La double contrainte de la situation d'enquête et le choix entre se taire ou dire « n'importe quoi »}

Le schéma précédent exclut une partie des contraintes que la situation d'enquête comme toute autre interaction sociale impose aux participants. À partir du moment où la personne sélectionnée accepte de participer à l'enquête, elle doit faire face à deux niveaux d'exigence développés par son engagement dans la situation; elle doit tendre à se conduire de façon à garder aussi bien sa propre face que celle de l'enquêteur. Goffman nomme « figuration » tout ce qu'entreprend une personne pour que ses actions ne fassent perdre la face à personne (y compris elle-même) (Goffman 1974). La réalisation dramatique que l'acteur fournit peut l'amener à supprimer la réplique qu'il voulait donner dans la mesure où celle-ci risquerait d'être incomprise du public et de lui aliéner sa sympathie. Il lui reste le choix entre inventer une autre réplique et sembler avoir un «trou » de mémoire. Les deux éléments de ce dilemme se taire ou dire n'importe quoi (par rapport à son « vrai » scénario) - ne sont pas équivalents du point de vue de l'interaction. La non-réponse dans le jeu d'un acteur peut créer un embarras puisqu'elle peut être interprétée comme un désengagement ${ }^{11}$ ou un manque de coopération. Ne pas répondre risque de porter atteinte à son image de « bonne » personne interrogée. Après avoir accepté de participer à l'enquête, laisser un blanc dans l'échange des questions-réponses peut vouloir signifier soit une remise en cause des questions formulées par l'enquêteur, donc de l'enquêteur lui-même, soit un détachement personnel traduisant une incapacité à tenir ses engagements. Bref, au moment de choisir dans le stock des réponses possibles, la personne interrogée doit s'accommoder pour le mieux des contraintes associées au fait d'être obligé de faire bonne figure non seulement en tant que personne revendiquant telle identité sociale, mais également en tant que personne impliquée dans une interaction.

La conciliation entre ces deux exigences a des effets sur le choix que les sujets interrogés effectuent dans leur répertoire figuratif. Les procédures d'évitement (partiel) que peut mettre en œuvre un sujet quand il doit indiquer un comportement, une opinion, un sentiment où il risque de perdre la face s'opposent donc entre elles : la non-réponse est préférable à la « vraie » réponse dans la mesure où elle fait disparaître le danger de donner une mauvaise image de soi (qui peut être paradoxalement une trop bonne dans le cas d'une enquête sur le patrimoine par exemple), mais elle offre l'inconvénient par rapport au choix d'une réponse « conforme » (ou supposée telle) de constituer un écart aux normes de comportement de la personne située dans une interaction. Il en découle un nouveau schéma pour la production d'une réponse. Un sujet interrogé dont l'état personnel coïncide avec la représentation qu'il a de la «mauvaise » réponse - mauvaise au sens de réponse menaçant l'image que le sujet veut donner de lui-même — a trois stratégies possibles pour répondre :
10 De nombreuses questions d'opinion ou de connaissance sont mixtes (a,b). Par exemple, la question « comment notez-vous tel ministre? » présuppose que la personne interrogée connaisse ce ministre. Certains sujets ignorants préfèrent fournir une note, sachant en réalité que la supercherie est indécelable, sauf quand un institut - comme l'EMNID en RFA en janvier 1982 - glisse le nom d'un ministre qui n'existe pas et recueille pour lui un bon score - la sixième place sur seize.

11 Sur l'engagement, voir « Involment » dans (Goffman, 1981). 


\section{Répertoire figuratif et classes sociales}

La situation d'enquête, même parfaitement standardisée, ne contraint pas tous les individus interrogés à une ligne de conduite identique. Elle oblige ces personnes à aborder des thèmes qui leur sont inégalement familiers et inégalement objets d'une conversation. Par exemple, la maîtrise de la situation d'une enquête sur les comportements sexuels dépend de la fréquence avec laquelle la personne a l'habitude de parler de ses relations ou problèmes sexuels. Le rapport entre le pourcentage des réponses « jamais » et celui des réponses « souvent » à la question « vous arrive-t-il de parler de vos problèmes sexuels? » constitue un moyen d'approcher, par l'inégale constitution de ce thème en sujet de conversation, l'inégale maîtrise de la situation d'enquête sur ce thème. Les femmes de plus de cinquante ans et avec un niveau scolaire primaire obtiennent un indice 10 et les hommes de moins de cinquante ans et avec un niveau scolaire secondaire ou supérieur un indice $0,1^{14}$. Lassurance (au sens goffmanien, c'est-à-dire «l'aptitude à supprimer toute tendance à baisser la tête lors des rencontres avec les autres » (Goffman, 1974)) des premières dans une situation où répondre est un devoir ne peut être qu'inférieure à celle des seconds. Quand la question porte sur un thème relevant d' « une réserve d'information », la personne interrogée perçoit un tel questionnement comme une violation de soi et perd son aisance.

En sociologie les enquêtes sont fréquemment centrées sur le rapport des individus et des groupes à la culture « légitime » - la visite d'un musée, l'écoute de la musique classique... Cette problématique «légitimiste » en sociologie provoque une structuration des questionnaires et au-delà des effets dans leurs conditions de réception ${ }^{15}$. Quand les personnes soumises à un interrogatoire sur leurs pratiques correspondant à la culture légitime ont un faible niveau d'appropriation de cette culture, elles sont contraintes de devoir choisir souvent les réponses qui risquent de leur faire perdre la face et d'avoir conscience de la perdre (le paradoxe de la légitimité culturelle étant que sa reconnaissance est intériorisée indépendamment de sa connaissance). L'authenticité inégale des personnes interrogées ne renvoie pas à une répartition inégale de l'hypocrisie ou d'une composante psychologique mais au sens différent que prend la situation d'enquête selon la position sociale ou culturelle de ces personnes. Ce que Pierre Bourdieu nomme la bonne volonté culturelle (Bourdieu, 1979) manifeste ce souci des individus dominés de retenir dans leur répertoire figuratif les réponses qui favorisent la meilleure image possible d'eux-mêmes dans ce contexte précis.

Cette bonne volonté peut entraîner une posture particulière pendant toute la durée du questionnement: le choix systématique de la même réponse (présumée la bonne). Nicole Tabard dans «Refus et approbation systématiques dans les enquêtes par sondage » (Tabard, 1975) a remarqué en effet que certaines personnes répondaient toujours « oui » indépendamment du sens des questions ${ }^{16}$. L'incohérence idéologique des réponses masque une cohérence de l'attitude vis-à-vis de l'enquête. Le portrait de ces femmes ayant un haut niveau d'approbation comporte les traits suivants : « être bénéficiaire de prestations sociales, être en contact avec les assistantes sociales, être en situation difficile d'indépendance économique et sociale ». Dépendantes des services sociaux, ces femmes adoptent face aux enquêtrices appartenant à ces services l'attitude qui leur apparaît être celle que les enquêtrices sont en droit d'attendre d'elles : la manifestation d'une coopération sans limites. Quel que soit leur état personnel, ces femmes retiennent dans le stock des réponses disponibles celles qui signifient qu'elles sont douées malgré leurs handicaps sociaux d'une qualité au moins, la déférence.
14 Exploitation secondaire des données de (Simon, 1972).

15 Les effets de cette problématique légitimiste sur la connaissance de la culture populaire sont traités par (Grignon et Passeron, 1982).

16 À la manière de Monsieur Songe achetant un poisson : « Le poissonnier lui demande :

- Je vous le prépare?

- Oui.

— Je vous laisse la tête?

- Oui.

— Et la laitance?

- Oui, et le boyau.

— Vous mangez le boyau?

— On ne sait jamais, c'est peut-être sain...

- Alors pourquoi me demander de préparer ce poisson?

— Je ne sais pas...

Et Monsieur Songe emporte son poisson en se disant que les commerçants d'aujourd'hui ne sont guère aimables. Il répondait "oui” à tout hasard, comme ça, pour faire plaisir... » (Pinget, 1982). 
Prendre le risque (apparent) de donner une mauvaise image de soi au cours d'une enquête est en revanche plus fréquemment le fait d'individus qui disposent de richesses sociales suffisantes pour que telle ou telle réponse n'altère pas leur présentation. Dans les enquêtes sur l'écoute de la télévision certains évitent de signaler qu'ils regardent des émissions comme «Au théâtre ce soir ». Au contraire, Claude Lévi-Strauss revendique publiquement le fait d'être un spectateur de cette émission théâtrale, il ajoute même que c'est une des rares émissions qu'il visionne, étant donné le temps qu'il réserve à la lecture. Cette congruence entre état personnel et choix dans le répertoire figuratif est rendue possible par les représentations antérieures que le public a de celui qui se permet un tel choix.

Le danger est d'autant moins grand pour l'auteur de la réponse que, sous les apparences du dérèglement, la logique culturelle est respectée : la hiérarchie des médias préservée avec le primat du livre sur la télévision et la définition illégitime de la télévision, médium spécialisé dans le divertissement ${ }^{17}$. L'authenticité dans la situation de l'enquête constitue un luxe relationnel.

\section{Le questionnaire entreprise « totalitaire » et la cohérence de l'expression}

À l'exception du Panoptique ou d'une institution totalitaire, l'acteur social a la possibilité de séparer ses différents publics de sorte que ceux qui «le voient dans l'un de ses rôles ne le voient pas dans un autre » (Goffman, 1968; 1973). Cette ségrégation des publics n'est pas la seule que forme l'individu, il réalise ou peut réaliser également une ségrégation de ses opinions personnelles ${ }^{18}$.

La logique d'un questionnaire qui essaie de saisir un ensemble de comportements et d'attitudes aussi diversifiées que possible pour permettre la construction théorique de l'espace des pratiques sociales est une entreprise totalitaire dans la mesure où elle contraint l'individu interrogé à s'exprimer sur des éléments de soi qui n'appartiennent pas habituellement à la même région. Toute mise en scène exige un certain degré de cohérence dans l'expression afin qu'une « fausse note » ne provoque pas une « rupture de ton » qui modifierait la représentation tout entière. La mise en scène à laquelle doit procéder un individu soumis à un tel questionnaire est encore plus difficile, la situation d'enquête augmentant le degré de cohérence expressive exigé pour la personne interrogée. Elle la contraint par le décloisonnement opéré par la « richesse » du questionnaire à se percevoir éventuellement en contradiction avec elle-même et donc à surveiller très attentivement le déroulement de l'enquête pour ne pas laisser filtrer les défaillances de sa cohérence interne. Ainsi pour ne pas avouer et s'avouer l'incompatibilité entre une affiliation politique de principe et certaines prises de position - incompatibilité rendue visible par le jeu du questionnement qui rapproche temporairement des opinions séparées - un individu est obligé de taire les opinions qui pourraient mettre un doute sur un des éléments de l'identité qu'il revendique ${ }^{19}$. Plutôt que d'avouer une position contraire au Parti Communiste par rapport à l'Afghanistan, une fraction des électeurs communistes se réfugient dans le silence; plutôt que de reconnaître leur accord avec le mot d'ordre d'un autre parti, une part des électeurs socialistes s'abstient de répondre ${ }^{20}$.

Le questionnaire menace l'unicité symbolique mise en scène par la personne interrogée ${ }^{21}$, brisant par la systématicité des thèmes abordés les frontières des comportements régionaux de cet acteur. Ainsi un adolescent peut très bien se définir comme différent de ses parents et leur emprunter certains traits, opinions, attitudes. Le questionner d'abord sur les caractéristiques de ses parents et ensuite sur les siennes le contraint pour préserver
17 Sur les stéréotypes du Livre et de la Télévision dans les classes supérieures, voir Passeron et al. (1981). Le dicton « faute avouée, faute pardonnée » s'applique davantage aux saints qu'aux pêcheurs. La croyance et la pratique régulière de la culture légitime autorisent bien des aveux, comme celui d'un ministre qui déclare à propos du livre Les caractères originaux de l'histoire rurale française qui figure dans sa bibliothèque : « celui-là, je l'ai volé. Je ne sais pas si c'est bien de raconter ça, après on va dire que je suis un voleur... J'avais 18 ans et j'aimais beaucoup Marc Bloch. Comme d'habitude dans ces cas-là, je voulais lire et avoir chez moi absolument tout ce qu'il avait écrit. La plupart de ses livres étaient disponibles en librairie, sauf celui-ci qui était introuvable. Alors je suis allé à la Bibliothèque Sainte-Geneviève et je l'ai volé » (Conan, « Dans la bibliothèque de J. Ralite », Libération, 30 juillet 1982). L'aveu d'une «fauche» de livres est d'autant plus aisé qu'il s'effectue dans un espace Libération — indulgent.

18 Contrairement au postulat de la psychologie sociale, selon lequel l'individu vit en permanence en « unité interne ». Voir par exemple Leonard (1972).

19 Inversement quand un individu revendique quelque chose d'inhabituel par rapport aux normes traditionnelles, il peut en « rajouter » pour montrer la force de sa détermination. C'est ainsi que les femmes mariées à des cadres supérieurs marquent leur rupture avec le modèle du monopole masculin de l'expression publique des opinions politiques. Elles indiquent en effet davantage leur intention de vote que les hommes cadres supérieurs alors que les autres femmes ont un taux de non-réponse plus élevé que les hommes.

20 Les rapports entre le taux de non-réponse des électeurs communistes et celui des électeurs socialistes sont 1,24 et 0,48 pour la question sur l'Afghanistan et la question sur l'installation de fusées nucléaires américaines en Europe (sondage Louis Harris, Le Matin, 24 janvier 1980).

21 Il s'agit de la cohérence vécue par l'acteur et non de celle que peut reconstruire le sociologue sous la forme par exemple des schèmes de l'habitus. 
l'image publique de son indétermination à taire ses opinions quand elles sont identiques à celles de ses parents. $56 \%$ des étudiants interrogés dans cet ordre déclarent ne pas avoir de préférence politique contre $33 \%$ des étudiants interrogés dans l'ordre inverse (opinions personnelles puis parentales) (Willick et Ashley, 1971). Le silence figuratif forme refuge pour le sujet quand il ressent les défaillances de sa mise en scène.

\section{Le pari empirique}

La connaissance antérieure de l'enquêteur ou plus précisément de l'institution dont se recommande l'enquêteur rend possibles les manœuvres pour déjouer les risques associés à l'interrogatoire ${ }^{22}$. Les personnes interrogées ne peuvent pas cependant, une fois qu'elles ont accepté le principe de l'enquête, enrayer le processus des «questions-réponses », la stratégie des héros de La guerre des boutons (Pergaud, 1963) leur devient interdite. Ceux-ci, ayant remarqué que leur maître était mal à l'aise dans le domaine sexuel, utilisent ce terrain pour empêcher toute poursuite de l'interrogatoire. Deux enfants manquent l'école pour préparer une bataille, leurs camarades préviennent le maître que le premier est « resté chez lui pour assister une vache en train de vêler tandis que l'autre mène au bouc une cabe qui s'obstine à ne pas... prendre ». Quand les deux retardataires arrivent, ils donnent les motifs de leur absence : «Monsieur, je viens vous dire que mon père m'a dit de vous dire que j'ai pas pu venir ce matin à l'école passe que j'ai mené not'cabe... - C'est bon, c'est bon, je sais, interrompit le père Simon qui n'aimait pas voir ses élèves se complaire à ces sortes de descriptions... Ça va bien! ça va bien, répondit-il de même et d'avance à Camus qui s'approchait. »

Il reste donc aux personnes interrogées à faire face aux contraintes de la situation dans laquelle elles sont engagées tout en essayant de faire bonne figure, aussi puisent-elles dans leur répertoire figuratif les types qui leur permettront de concilier les exigences de l'interaction - la coopération - et de la mise en scène. C'est pourquoi répondre «n'importe quoi » ou «bien » répondre est plus fréquent que le silence, cela donne le change à l'enquêteur et fournit l'occasion de réaliser une présentation de soi conforme et positive.

Ces remarques cernent le pari de la sociologie empirique : la vérité de la condition sociale d'un agent (ou d'un groupe) peut être appréhendée au travers des représentations théâtrales qu'il fournit (ou des représentations mentales qu'autrui donne de lui ${ }^{23}$. La détruisent-ils ? Les sociologues et les statisticiens peuvent recourir à trois moyens pour la gagner malgré la figuration - inévitable - du public.

Le premier, le raffinement méthodologique, consiste en une amélioration des techniques du questionnaire ${ }^{24}$ notamment en proposant dans la formulation des questions une alternative complète des réponses, un stock suffisant. Ainsi, dans une enquête sur les femmes au foyer et leur « désir» d'activité professionnelle, on posait :

- soit la question (a) « aimeriez-vous avoir un emploi si cela était possible?»,

soit la question (b) «préféreriez-vous avoir un emploi ou préférez-vous ne vous occuper que de votre foyer?»
22 Quels effets produisent les images respectives de l'Insee, du CNRS, de l'Université lors de la passation d'un questionnaire?

23 Sur les représentations que les employeurs ont de leurs salariés, voir Baudelot (1981), Eymard-Duvernay (1981), et sur les représentations que les conjoints ont de leurs partenaires, voir Thévenot (1981).

24 La revue Public Opinion Quartely représente ce courant. Michel

Grumbach a réalisé une synthèse des études publiées entre 1970 et 1980 dans cette revue (Grumbach, 1982). 
Les pourcentages des réponses varient fortement :

\begin{tabular}{|l|l|l|}
\hline \multirow{2}{*}{ Réponses } & Questions & $\mathrm{b}$ \\
\cline { 2 - 3 } & $\mathrm{a}$ & $10 \%$ \\
\hline «avoir un emploi » & $17 \%$ & $14 \%$ \\
\hline «avoir un emploi à mi-temps & $38 \%$ & $68 \%$ \\
\hline $\begin{array}{l}\text { «préfèrent s'occuper de leur } \\
\text { foyer» }\end{array}$ & $19 \%$ & $8 \%$ \\
\hline sans opinion & $26 \%$ & $8 \%$ \\
\hline
\end{tabular}

Quand la formulation de la question semble n'envisager que l'activité professionnelle, les femmes au foyer se réfugient dans le silence ou choisissent la réponse du travail à mi-temps, « mi-chèvre, mi-chou », qui à la fois se situe dans la perspective dominante de la nécessité d'une activité professionnelle et dans la défense de leur statut.

Avec le second moyen, la ruse méthodologique, de nouvelles situations d'enquête sont inventées, quelquefois à l'intérieur même de la situation classique. C'est William Labov qui tente dans Sociolinguistique de résoudre «le paradoxe de l'observateur » — « découvrir comment les gens parlent quand on ne les observe pas systématiquement, mais la seule façon d'y parvenir est de les observer systématiquement » - en construisant des procédés de recueil de discours « familier », ordinaire (Labov, 1976a; 1976b). La question reste cependant ouverte de savoir si ces procédés de saisie d'une mise en scène plus quotidienne sont compatibles avec les contraintes du recueil des données statistiques.

Enfin, un troisième moyen, « l'exigence renforcée de comparaison », est proposé notamment par Jean-Claude Passeron (1982).

«L'antidote se trouve dans le refus des enfermements technologiques » et dans l'analyse comparative « de vastes ensembles d'observations culturelles et historiques » qui seule peut permettre « d'ordonner au moins approximativement un univers de variations dans lequel paroles et non-paroles ne veulent jamais dire la même chose, selon les époques, les groupes, les situations de parole, les formes de questionnement et les objets de discours ».

L'analyse des « éléments rituels inhérents aux interactions sociales » (Goffman, 1974) montre quand elle est appliquée à la situation d'enquête l'importance de la phase de recueil des données. La « sociologie des circonstances » ne doit-elle pas devenir partie constituante des travaux de recherche empirique? 


\section{Bibliographie}

BAUDELOT Christian, 1981, « Mesure de la mobilité et chiffrement de la CSP dans les déclarations annuelles de salaires », in CoLL., Les CSP et leur repérage dans les enquêtes, INSEE, coll. Archives et Documents $n^{\circ} 38$, p. 167-200

Boltanski Luc et MALDIDIER Pascale, 1977, La vulgarisation scientifique et son public, Paris, CSE, rapport CORDES.

Bourdieu Pierre, 1979, La Distinction, Paris, Minuit.

Bradburn Norman M., Sudman Seymour, Blair Ed et Stocking Carol, 1978, «Question threat and response bias », Public Opinion Quartely, vol. $42, \mathrm{n}^{\circ} 2$, p. 221-234.

DE SINGLY François, 1982, « La gestion sociale des silences », Consommation, $\mathrm{n}^{\circ}$ 4, p. 37-63.

DESPORTES Jean-Pierre, 1975, Les effets de la présence de l'expérimentateur dans les sciences du comportement, Paris, Éditions du CNRS.

EYMARD-DUVERnAY François, 1981, « Qualification, poste, salaire », in ColL., Les CSP et leur repérage dans les enquêtes, Insee, coll. Archives et Documents, $\mathrm{n}^{\circ}$ 38, p. 83-103

FAVRET-SAADA Jeanne, 1981, Corps pour corps, Paris, Gallimard.

FoucAult Michel, 1976, La volonté de savoir, Paris, Gallimard.

GAXIE Daniel, Le sens caché, Paris, Seuil.

Goffman Erving, 1968, Asiles, Paris, Minuit.

GofFMAN Erving, 1973, La présentation de soi, Paris, Minuit.

Goffman Erving, 1974, Rites d'interaction, Paris, Éditions de Minuit.

GofFMAN Erving, 1981, « Engagement », in Winkin Yves (éd.), La nouvelle communication, Paris, Seuil, p. 267-278.

Grignon Claude et PASSERon Jean-Claude, 1982, « Sur la culture populaire », Paris, séminaire de l'EHESS.

GRUMBACH Michel, 1982, «Sur la méthodologie du questionnaire», ronéo. Habermas Jürgen, 1978, L'espace public, Paris, Payot.

LABOv William, 1976a, «L'étude de la langue dans son contexte social », in Labov William, Sociolinguistique, Paris, Minuit, p. 127-174.

LABOv William, 1976b, « Le dégagement des styles conceptuels », in LABOv William, Sociolinguistique, Paris, Minuit, p. 257-351.

LECLERC Gérard, 1979, L'observation de l'homme, Paris, Seuil.

LÉONARD François, 1972, «Un modèle du sujet ; l'équilibre de Heider », in Moscovici Serge (dir.), Introduction à la psychologie sociale, tome 1, Paris, Larousse, p. 103-138.

Passeron Jean-Claude, 1982, « Le langage de l'abstention », Consommation, $\mathrm{n}^{\circ} 4$, p. 3-11.

Passeron Jean-Claude, Grumbach Michel, Bénard Monique, Kadri Aïssa, Martinon Jean-Pierre, Naffrechoux Martine, Parmentier Patrick, PorTo-VAsQuez Fernando et DE Singly François, 1981, L'OEil à la page, Paris, Rapport pour le Ministère de la Culture, GIDES, $2^{\mathrm{e}}$ édition.

Pergaud Louis, 1963, La guerre des boutons, Paris, Mercure de France.

PINGET Robert, 1982, Monsieur Songe, Paris, Minuit.

SACKs Harvey, 1973, « Tout le monde doit mentir », Communications, $\mathrm{n}^{0} 20$, p. 182-203.

SCHWARTz Richard D. et Skolnick Jerome E., 1962, « A study of legal stigma », Social Problems, $\mathrm{n}^{\circ}$ 10, p. 133-138.

Simon Pierre, Mironer Lucien, Gondonneau Jean et Dourlen-Rollier Anne-Marie, 1972, Rapport sur le comportement sexuel des Français, Paris, Julliard-Charron. 
TABARD Nicole, 1975, « Refus et approbation systématiques dans les enquêtes par sondage », Consommation, ${ }^{\circ}$ 4, p. 59-76.

THÉVENot Laurent, 1981, « Un emploi à quel titre? », in ColL., Les CSP et leur repérage dans les enquêtes, INSEE, coll. Archives et Documents, $\mathrm{n}^{\circ}$ 38, p. 9-39.

Willick Daniel H. et Ashley Richard K., 1971, « Survey question order and the political party preferences of college students and their parents », Public Opinion Quartely, vol. 35, nº 2, p. 189-199. 